\title{
A Note on Subgeometric Rate Convergence for Ergodic Markov Chains in the Wasserstein Metric
}

\author{
Mokaedi V. Lekgari \\ Mathematics Department, University of Botswana, P/Bag 0022 Gaborone, Botswana \\ lekgarimv@mopipi.ub.bw
}

Keywords: Markov Chains; Ergodicity; Wasserstein metric.

\begin{abstract}
We investigate subgeometric rate ergodicity for Markov chains in the Wasserstein metric and show that the finiteness of the expectation $E_{(i, j)}\left[\sum_{k=0}^{\tau_{\Delta}-1} r(k)\right]$, where $\tau_{\Delta}$ is the hitting time on the coupling set $\Delta$ and $r$ is a subgeometric rate function, is equivalent to a sequence of Foster-Lyapunov drift conditions which imply subgeometric convergence in the Wassertein distance. We give an example for a 'family of nested drift conditions'.
\end{abstract}

\section{Introduction and Notations}

We start with a brief review of ergodicity. Let $\mathbb{Z}_{+}=\{0,1,2, \ldots\}, \mathbb{N}_{+}=\{1,2, \ldots\}$, and $\mathbb{R}_{+}=[0, \infty)$. Let $\left(\Phi_{n}\right)_{n \in \mathbb{Z}_{+}}$denote a Markov chain with transition kernel $P$ on a countably generated state space denoted by $(\mathcal{X}, \mathcal{B}(\mathcal{X})) \cdot P^{n}(i, j)=P_{i}\left(\Phi_{n=j}\right)=E_{i}\left[\mathbf{1}_{\Phi_{n=j}}\right]$, where $P_{i}$ and $E_{i}$ respectively denote the probability and expectation of the chain under the condition that its initial state $\Phi_{0}=i$, and $\mathbf{1}_{A}$ is the indicator function of set $A$. According to Markov's theorem, a Markov chain $\left(\Phi_{n}\right)_{n \in \mathbb{Z}_{+}}$is ergodic if there's positive probability to pass from any state, say $i \in \mathcal{X}$ to any other state, say $\cdot \in \mathcal{X}$ in one step. That is, for states $i, \cdot \in \mathcal{X}$ then chain $\left(\Phi_{n}\right)_{n \in \mathbb{Z}_{+}}$is ergodic if $P^{1}(i, \cdot)>0$.

Also the chain $\left(\Phi_{n}\right)_{n \in \mathbb{Z}_{+}}$is said to be (ordinary) ergodic if $\forall i, \cdot \in \mathcal{X}$ then

$$
P^{n}(i, \cdot) \rightarrow \pi(\cdot) \text { as } n \rightarrow \infty
$$

where the $\sigma$-finite measure $\pi$ is the invariant limit distribution of the chain.

Chain $\left(\Phi_{n}\right)_{n \in \mathbb{Z}_{+}}$is referred to as geometrically ergodic if there exists some measurable function $V: \mathcal{X} \rightarrow(0, \infty)$, and constants $\beta<1$ and $M<\infty$ such that

$$
\left\|P^{n}(i, \cdot)-\pi(\cdot)\right\| \leq M V(i) \beta^{n}, \quad \forall n \in \mathbb{N}_{+},
$$

where here and hereafter for the (signed) measure $\mu$ we define $\mu(f)=\int \mu(d j) f(j)$, and the norm $\|\mu\|$ is defined by $\sup _{|g| \leq f}|\mu(g)|$, whereas the total variation norm is defined similarly but with $f \equiv 1$.

Markov chain $\left(\Phi_{n}\right)_{n \in \mathbb{Z}_{+}}$is strongly ergodic if

$$
\lim _{n \rightarrow \infty} \sup _{i \in \mathcal{X}}\left\|P^{n}(i, \cdot)-\pi(\cdot)\right\|=0 .
$$

Loosely speaking subgeometric ergodicity, which we define next, is a kind of convergence that's faster than ordinary ergodicity but slower than geometric ergodicity.

Let function $r \in \Lambda_{0}$ where $\Lambda_{0}$ is the family of measurable increasing functions $r: \mathbb{R}_{+} \rightarrow[1, \infty)$ satisfying $\frac{\log r(t)}{t} \downarrow 0$ as $t \uparrow \infty$. Let $\Lambda$ denote the class of positive functions $\bar{r}: \mathbb{R}_{+} \rightarrow(0, \infty)$ such that for some $r \in \Lambda_{0}$ we have;

$$
0<\lim _{n} \inf \frac{\bar{r}(n)}{r(n)} \leq \lim _{n} \sup \frac{\bar{r}(n)}{r(n)}<\infty
$$

Indeed (1) implies the equivalence of the class of functions $\Lambda_{0}$ with the class of functions $\Lambda$. Examples of functions in the class $r \in \Lambda$ is the rate $r(n)=\exp \left(s n^{1 /(1+\alpha)}\right), \alpha>0, s>0$. Without loss to 
generality we suppose that $r(0)=1$ whenever $r \in \Lambda$. The properties of $r \in \Lambda_{0}$ which follow from (1) and are to be used frequently in this study are;

$$
\begin{gathered}
r(x+y) \leq r(x) r(y) \forall x, y \in \mathbb{R}_{+} \\
\frac{r(x+a)}{r(x)} \rightarrow 1 \text { as } x \rightarrow \infty, \text { for each } a \in \mathbb{R}_{+} .
\end{gathered}
$$

$\Lambda$ is referred to as the class of subgeometric rate functions(cf. [3]).

Let $r \in \Lambda$, then the ergodic chain $\Phi_{n}$ is said to be subgeometrically ergodic of order $r$ in the $f$ norm, (or simply $(f, r)$-ergodic) if for the unique invariant distribution $\pi$ of the process and $\forall i \in \mathcal{X}$, then

$$
\lim _{n \rightarrow+\infty} r(n)\left\|P^{n}(i, \cdot)-\pi(\cdot)\right\|_{f}=0,
$$

where $\|\sigma\|_{f}=\sup _{|g| \leq f}|\sigma(g)|$ and $f: \mathcal{X} \rightarrow[1, \infty)$ is a measurable function. Also for subgeometric ergodic to hold it's necessary that there exist a deterministic sequence $\left\{V_{n}\right\}$ of functions $V_{n}: \mathcal{X} \rightarrow$ $[1, \infty)$ which satisfy the Foster-Lyapunov drift condition:

$$
P V_{n+1} \leq V_{n}-r(n) f+b r(n) \mathbf{1}_{C}, n \in \mathbb{Z}_{+} .
$$

for a petite set $C \in \mathcal{B}(\mathcal{X})$ and a constant $b \in \mathbb{R}_{+}$such that $\sup _{C} V_{0}<\infty$. The Foster-Lyapunov drift conditions provide bounds on the return time to accessible sets thereby availing some control on the Markov process dynamics by focusing on the hitting times on a particular set.

Convergence in the Wasserstein distance is a very interesting research area through which [1] amongst other authors suggested a new technique for establishing subgeometric ergodicity. Following [1] we define the Wasserstein distance as follows. Let $(\mathcal{X}, d)$ be a Polish space where $d$ is a distance bounded by 1 and let $\mathcal{P}(\mathcal{X})$ denote the set of all probability measures on state space $(\mathcal{X}, \mathcal{B}(\mathcal{X}))$. Let $\mu, \nu \in \mathcal{P}(\mathcal{X}) ; \lambda$ is a coupling of $\mu$ and $\nu$ if $\lambda$ is a probability on the product space $(\mathcal{X} \times \mathcal{X}, \mathcal{B}(\mathcal{X} \times \mathcal{X}))$, such that $\lambda(A \times \mathcal{X})=\mu(A)$ and $\lambda(\mathcal{X} \times A)=\nu(A) \forall A \in \mathcal{B}(\mathcal{X})$. We further let $\mathcal{C}(\mu, \nu)$ be set of all probability measures on $(\mathcal{X} \times \mathcal{X}, \mathcal{B}(\mathcal{X} \times \mathcal{X}))$ with marginals $\mu$ and $\nu$, and $Q$ be the coupling Markov kernel on $(\mathcal{X} \times \mathcal{X}, \mathcal{B}(\mathcal{X} \times \mathcal{X}))$ such that for every $i, j \in \mathcal{X}$, then $Q((i, j), \cdot)$ is a coupling of $P(i, \cdot)$ and $P(j, \cdot)$. The Wasserstein metric associated with the semimetric $d$ on $\mathcal{X}$, between two probability measures $\mu$ and $\nu$, is then given as

$$
W_{d}(\mu, \nu):=\inf _{\gamma \in \mathcal{C}(\mu, \nu)} \int_{\mathcal{X} \times \mathcal{X}} d(i, j) d \gamma(i, j) .
$$

When $d$ is the trivial metric $d_{0}(i, j)=\mathbf{1}_{i \neq j}$, then the associated Wasserstein metric is the total variation metric $W_{d_{0}}(\mu, \nu)=d_{T V}(\mu, \nu):=2 \sup _{C \in \mathcal{B}(\mathcal{X})}|\mu(C)-\nu(C)|, \mu, \nu \in \mathcal{P}(\mathcal{X})$.

A set $C$ is said to be small if there exists a constant $\epsilon>0$ such that for all $i, j \in C$ then $\frac{1}{2} d_{T V}(P(i, \cdot), P(j, \cdot)) \leq 1-\epsilon$. Set $C \in \mathcal{B}(\mathcal{X})$ is petite if there exist some non-trivial measure $\nu_{a}$ on $\mathcal{B}(\mathcal{X})$ and some probability distribution $a=\left\{a_{n}: n \in \mathbb{Z}_{+}\right\}$such that

$$
\sum_{n=1}^{\infty} a_{n} P^{n}(x, \cdot) \geq \nu_{a}(\cdot), \quad \forall x \in C .
$$

Petite sets generalize small sets. The first hitting time on small set $C$ delayed by a constant $\delta>0$ is given by $\tau_{C}^{\delta}=\inf \left\{n \geq \delta: \Phi_{n} \in C\right\}$. We also have $\tau_{C}^{+}=\inf \left\{n \geq J_{1}: \Phi_{n} \in C\right\}$ as the first hitting time on the set $C$ after the first jump $J_{1}$ of the process. We note that $\xi_{C}^{+}=\xi_{C}$ if $\Phi_{0} \notin C$. In the case when $\delta=0$ we have $\tau_{C}^{0}=\tau_{C}$. If $C$ is a singleton consisting only of state $i$ then we write $\tau_{i}^{\delta}$ for $\tau_{C}^{\delta}$ and equivalently $\tau_{i}^{+}$for $\tau_{C}^{+}$. It's worth noting that finite mean return times $E_{i}\left[\tau_{i}^{+}\right]<\infty$ guarantee ergodicity or the existence of stationary probability and the convergence $P^{n}(i, j)-\pi \rightarrow 0$ 
as $n \rightarrow \infty$. It's also known that subgeometric ergodic is equivalent to $(f, r)$-regularity. We define $(f, r)$-regularity as follows. Set $C \in \mathcal{X}$ is said to be $(f, r)$-regular if for all $i \in C$, a measurable function $f: \mathcal{X} \rightarrow[1, \infty)$, rate function $r$ and $\forall B \in \mathcal{B}^{+}(\mathcal{X})$ then,

$$
\sup _{i \in C} E_{i}\left[\sum_{k=0}^{\tau_{B}-1} r(k) f\left(\Phi_{k}\right)\right]<\infty
$$

where the set $\mathcal{B}^{+}(\mathcal{X})$ is set of all accessible(or $\Psi$-irreducible) sets. By finding a suitable contracting metric $d$ which may be different from the discrete metric, and a suitable Foster-Lyapunov function $V$ with a ' $d$-small' sublevel set, [1] suggested a new technique for establishing subgeometric ergodicity. Then [2] extended the results of [1] by establishing sufficient conditions for the existence of the invariant distribution and subgeometric rates of convergence for chains that are not necessarily $\Psi$-irreducible. For the Polish space $\left(\mathcal{X}, d_{*}\right)$, the $d$-small set of [1] was extended by [2] to the $(\ell, \epsilon, d)$ coupling set(or simply coupling set) $\triangle \in \mathcal{X} \times \mathcal{X}$, where $\ell \in \mathbb{Z}_{+}, \epsilon \in(0,1)$, and $d$ is a distance on state space $\mathcal{X}$, topologically equivalent to $d_{*}$ and bounded by 1 .

Let $r \in \Lambda$, then we denote the sequence $R$ as

$$
R(n)=\sum_{k=0}^{n-1} r(k) n \in \mathbb{N}_{+}, R(0)=1 .
$$

We show, in this paper through Proposition 1, that the sequence of drift inequalities proposed by [2] hold if and only if $R\left(\tau_{\triangle}\right)<\infty$. As an example, we explore a 'family of nested drift conditions' as proposed by [4] in both the discrete and continuous cases whose results we transfer to the convergence in the Wasserstein metric through Proposition 3.

\section{Main Results}

\section{Lyapunov Drift Inequalities}

In light of the definitions and notations given above, we state Assumption A1 as follows:

A1. There exist a coupling set $\Delta \in \mathcal{B}(\mathcal{X} \times \mathcal{X})$ such that for a sequence $r \in \Lambda$ and $\forall i, j \in \mathcal{X}$,

$$
\sup _{(i, j) \in \Delta} E_{(i, j)}\left[R\left(\tau_{\triangle}\right)\right]<\infty
$$

According to Theorem 2.1(ii) of [5], as mentioned already, the Foster-Lyapunov drift conditions in (5) can also be used to define subgeometric rate ergodicity. Following this result [2] proposed a sequence of drift functions according to Assumption $\mathbf{A} 2$ as follows.

A2. There exist

1. a sequence of measurable functions $\left\{\mathcal{V}_{n}\right\} n \in \mathbb{Z}_{+}, \mathcal{V}_{n}: \mathcal{X} \times \mathcal{X} \rightarrow \mathbb{R}_{+}$,

2. a set $\Delta \in \mathcal{B}(\mathcal{X} \times \mathcal{X})$, a constant $b \in \mathbb{R}_{+}$and a sequence $r \in \Lambda$ such that $\forall i, j \in \mathcal{X}$ and for every coupling $\alpha \in \mathcal{C}(P(i, \cdot), P(j, \cdot))$;

$$
\int_{\mathcal{X} \times \mathcal{X}} \mathcal{V}_{n+1}(z, t) d \alpha(z, t) \leq \mathcal{V}_{n}-r(n) f+b r(n) \mathbf{1}_{(i, j) \in \Delta}, n \in \mathbb{Z}_{+} .
$$

Further, there exist measurable functions $\left(V_{n}\right) n \in \mathbb{Z}_{+}$such that $\forall i, j \in \mathcal{X}$ and any $n \in \mathbb{Z}_{+}$:

$$
\mathcal{V}_{n}(i, j) \leq V_{n}(i)+V_{n}(j) \text { and } P V_{n+1} \leq V_{n}+b r(n)
$$

and $\forall k \in \mathbb{Z}_{+}$

$$
\sup _{(i, j) \in \Delta}\left\{P^{k} V_{0}(i)+P^{k} V_{0}(j)\right\}<+\infty \text { and } \forall i \in \mathcal{X}, P^{k} V_{0}(i)<+\infty .
$$


Proposition 1. $A 1 \Leftrightarrow A 2$.

Proof. $\quad$ 1. $\mathbf{A} \mathbf{1} \Rightarrow \mathbf{A 2}$

Let $r \in \Lambda_{0}$ and $\left\{\mathcal{V}_{n}\right\},\left\{\mathcal{W}_{n}\right\}: \mathcal{X} \times \mathcal{X} \rightarrow[0, \infty)$ be sequences of functions defined for all $n \in \mathbb{Z}_{+}$by

$$
\begin{gathered}
\mathcal{V}_{n}=E_{i, j}\left[\sum_{k=1}^{\tau_{\Delta}} r(n+k)\right] \mathbf{1}_{\Delta^{c}}+r(n), \\
\mathcal{W}_{n}=E_{i, j}\left[\sum_{k=1}^{\tau_{\Delta}} r(n+k)\right] .
\end{gathered}
$$

Then by the submultiplicative property (2) and Assumption A1 we have $\mathcal{V}_{0} \leq \mathcal{V}_{n} \leq r(n) \mathcal{V}_{0}<$ $\infty$ and $\mathcal{W}_{0} \leq \mathcal{W}_{n} \leq r(n) \mathcal{W}_{0}<\infty$. We also note that $\mathcal{V}_{n}=\mathcal{W}_{n} \mathbf{1}_{\Delta^{c}}+r(n)$ such that

$$
\begin{aligned}
\int_{\mathcal{X} \times \mathcal{X}} \mathcal{V}_{n+1}(z, t) d \alpha(z, t) & =\int_{\mathcal{X} \times \mathcal{X}}\left(\mathcal{W}_{n+1} \mathbf{1}_{\Delta c}(z, t)+r(n+1)\right) d \alpha(z, t) \\
& \leq \int_{\mathcal{X} \times \mathcal{X}}\left(\mathcal{W}_{n+1} \mathbf{1}_{\Delta^{c}}(z, t)+\mathcal{W}_{n+1} \mathbf{1}_{\Delta}(z, t)\right) d \alpha(z, t) \\
& =\int_{\mathcal{X} \times \mathcal{X}}\left(\mathcal{W}_{n+1}(z, t)\right) d \alpha(z, t) \\
& \leq \mathcal{W}_{n}(i, j) \\
& =\mathcal{V}_{n}(i, j)-r(n)+\mathcal{W}_{n} \mathbf{1}_{\{(i, j) \in \Delta\}} \\
& \leq \mathcal{V}_{n}(i, j)-r(n)+r(n) \mathcal{W}_{n} \mathbf{1}_{\{(i, j) \in \Delta\}} \\
& \leq \mathcal{V}_{n}(i, j)-r(n)+r(n) \mathcal{W}_{0} \mathbf{1}_{\{(i, j) \in \Delta\}} \\
& \leq \mathcal{V}_{n}(i, j)-r(n)+\operatorname{br}(n) \mathbf{1}_{\Delta}(i, j),
\end{aligned}
$$

where we choose $b=\sup _{\triangle} \mathcal{W}_{0}$.

2. $\mathbf{A} 2 \Rightarrow \mathbf{A} 1$

Analogous to Proposition 11.3.3 in [7] we get from A2 that for some constant $c<\infty$

$$
E_{i, j}\left[R\left(\tau_{\Delta}\right)\right] \leq\left\{\begin{array}{l}
\mathcal{V}_{0}(i, j) \\
r(0)+c Q \mathcal{V}_{0}(i, j) \quad,(i, j) \in \mathcal{X} \times \mathcal{X}
\end{array}\right.
$$

Then by Eq. 8, Eq. 9 and $\sup _{\Delta} \mathcal{V}_{0}<\infty$ we get $E_{i, j}\left[R\left(\tau_{\Delta}\right)\right]<\infty$.

\section{Family of nested drift conditions}

The phenomenon of ergodicity as given in Proposition 1 is not altogether new as is clear from the following Proposition which deals with a family of nested drift conditions for subgeometrically ergodic general state space Markov processes analogous to one proposed by [4].

Proposition 2. Suppose that there are functions $\mathcal{V}_{k}, \mathcal{W}_{k}: \mathcal{X} \times \mathcal{X} \rightarrow[1, \infty)$, where $k \in \mathbb{Z}_{+}$, a coupling set $\triangle \in \mathcal{B}(\mathcal{X} \times \mathcal{X})$ such that for any initial state $(i, j) \in \mathcal{X} \times \mathcal{X}$ of the chain, we have

$$
E_{i, j}\left[\mathcal{V}_{k+n+1}(i+1, j+1) \mid \mathcal{F}_{n}\right] \leq \mathcal{V}_{k}(i, j)-E_{i, j}\left[R\left(\tau_{\Delta}\right)\right]+\mathcal{W}_{k}(i, j) \mathbf{1}_{(i, j) \in \Delta\}}
$$

then the chain $\Phi_{n}$ is subgeometrically ergodic. 
Proposition 2 follows from Proposition 3 which is the extension of Proposition 3.1 in [4] on family of nested drift conditions. As is evident in the Propositions that follow we note that the results of Proposition 2 stay the same if we replace $R\left(\tau_{\Delta}\right)$ with $R_{m}\left(\tau_{\Delta}\right)$, where $R_{m}(n)=\sum_{k=0}^{n-1} r(m+k)$ for $m \geq 0, n \geq 1$ with $R(0)=1$. The results also stay the same if we replace the measurable functions $\mathcal{V}_{k}$ and $\mathcal{W}_{k}$ with $V_{k}$ and $W_{k}$ respectively as is the case for convergence in the $f$-norm.

Proposition 3. Let the chain $\left(\Phi_{n}\right)_{n \in \mathbb{Z}_{+}}$be irreducible and aperiodic. Further suppose that there are functions $f, V_{k}, W_{k}: \mathcal{X} \rightarrow[1, \infty)$, where $k \in \mathbb{Z}_{+}$, with $\sup _{C} V_{k}<\infty$, $\sup _{C} W_{k}<\infty$ and a small set $C$ such that for a non-decreasing sequence of stopping times $\left\{\mathcal{T}_{n}: n \in \mathbb{Z}_{+}\right\}$and any $\Phi_{\mathcal{T}_{n}} \in \mathcal{X}$, we have

$$
E_{\Phi_{\mathcal{T}_{n}}}\left[V_{k+\mathcal{T}_{n+1}}\left(\Phi_{\mathcal{T}_{n+1}}\right) \mid \mathcal{F}_{n}\right] \leq V_{k}\left(\Phi_{\mathcal{T}_{n}}\right)-E_{\Phi_{\mathcal{T}_{n}}}\left[\sum_{l=\mathcal{T}_{n}}^{\mathcal{T}_{n+1}-1} f\left(\Phi_{l}\right) r(k+l)\right]+W_{k}\left(\Phi_{\mathcal{T}_{n}}\right) \mathbf{1}_{\left\{\Phi_{\mathcal{T}_{n}} \in C\right\}}
$$

then the chain $\Phi_{n}$ is $(f, r)$-ergodic.

Proof. We let $\mathcal{T}_{n}$ be some random stopping time with $\mathcal{F}_{n}$ as the $\sigma$-algebra of events generated by $\mathcal{T}_{n}$. Then by Dynkin's inequality we get

$$
E_{\Phi_{\mathcal{T}_{n}}}\left[\sum_{l=1}^{\mathcal{T}_{n}-1} f\left(\Phi_{l}\right) r(k+l)\right] \leq V_{k}\left(\Phi_{\mathcal{T}_{n}}\right)+W_{k}\left(\Phi_{\mathcal{T}_{n}}\right) \mathbf{1}_{\left\{\Phi_{\mathcal{T}_{n}} \in C\right\}}
$$

which implies that

$$
\begin{aligned}
E\left[\sum_{n=0}^{\tau_{C}-1} r(n) f(n) \mid \mathcal{F}_{n}\right] & \leq V_{0}(i)+W_{0}(i) E\left[\sum_{n=0}^{\tau_{C}-1} \mathbf{1}_{\{i \in C\}}\right] \\
& \leq c\left(V_{0}(i)+W_{0}(i)\right), \quad c \in[0, \infty) \\
& <\infty .
\end{aligned}
$$

by assuming that $\sup _{C} V_{k}<\infty, \sup _{C} W_{k}<\infty$. Hence the chain is $(f, r)$-ergodic.

Proposition 4. Let the chain $\left(\Phi_{t}\right)_{t \in \mathbb{R}_{+}}$be irreducible. Further suppose that there are functions $f, V_{k}$, $W_{k}: \mathcal{X} \rightarrow[1, \infty)$ where $k \in \mathbb{Z}_{+}$, some constant $\varepsilon>0$, a small set $C$ such that for a non-decreasing sequence of stopping times $\left\{\mathcal{T}_{n}: n \in \mathbb{Z}_{+}\right\}$and any $\Phi_{\mathcal{T}_{n}} \in \mathcal{X}$, we have

$$
E_{\Phi_{\mathcal{T}_{n}}}\left[V_{k+\mathcal{T}_{n+1}}\left(\Phi_{\mathcal{T}_{n+1}}\right) \mid \mathcal{F}_{n}\right] \leq V_{k}\left(\Phi_{\mathcal{T}_{n}}\right)-E_{\Phi_{\mathcal{T}_{n}}}\left[\int_{0}^{\varepsilon} f\left(\Phi_{s}\right) r(k+s) d s\right]+W_{k}\left(\Phi_{\mathcal{T}_{n}}\right) \mathbf{1}_{\left\{\Phi_{\mathcal{T}_{n}} \in C\right\}}
$$

then the chain $\Phi_{t}$ is $(f, r)$-ergodic.

Proof. Note that this Proposition is the continuous counterpart to Proposition 3. For the term

$$
E_{\Phi_{\mathcal{T}_{n}}}\left[\int_{0}^{\varepsilon} f\left(\Phi_{s}\right) r(k+s) d s\right]
$$

where $\varepsilon>0$, by the submultiplicative property (2) we have

$$
E_{\Phi_{\mathcal{T}_{n}}}\left[\int_{0}^{\varepsilon} f\left(\Phi_{s}\right) r(k+s) d s\right] \leq r(k) E_{\Phi_{\mathcal{T}_{n}}}\left[\int_{0}^{\varepsilon} f\left(\Phi_{s}\right) r(s) d s\right]<\infty
$$

because $r \in \Lambda$ is finite for all $k \in \mathbb{Z}_{+}$and $E_{\Phi_{\mathcal{T}_{n}}}\left[\int_{0}^{\varepsilon} f\left(\Phi_{s}\right) r(s) d s\right]<\infty$ by proof of Theorem 6 in [6], hence we conclude that the chain $\Phi_{t}$ is $(f, r)$-ergodic. 


\section{References}

[1] O. Butkovsky, Subgemetric rates of convergence of Markov processes in the Masserstein metric, Ann. Appl. Probab. 24(2) (2014) 526-552.

[2] A. Durmus, G. Fort, E. Moulines, New conditions for subgeometric rates of convergence in the Wasserstein distance for Markov chains, Unpublished paper, 2014. Available on: https://hal.archives-ouvertes.fr/hal-00948661v1/document.

[3] E. Nummelin, P. Tuominen, The rate of convergence in Orey's theorem for Harris recurrent Markov chains with applications to renewal theory, Stochastic Process Appl. 15 (1983) 295-311.

[4] S.B. Connor, G. Fort, State-dependent Foster-Lyapunov criteria for subgeometric convergence of Markov chains, Stochastic Processes and their Applications. 119 (2009) 4176-4193.

[5] S.P. Meyn, R.L. Tweedie, State-dependent criteria for convergence of Markov chains, Ann. Appl. Prob. (1994) 149-168.

[6] M.V. Lekgari, Subgeometric Ergodicity Analysis of Continuous-time Markov Chains Under Random-time State-dependent Lyapunov Drift Conditions, J. Prob. Stat. 2014 (2014), Article ID 274535.

[7] S.P. Meyn, R.L. Tweedie, Markov Chains and Stochastic Stability, Springer, 1993.

[8] R.L. Tweedie, Criteria for rates of convergence of Markov chains, in J.F.C. Kingman , G.E.H. Reuter(Eds.), Probaility Statistics and Analysis, in: London Mathematical Society Lecture Note Series, Cambridge University Press, 1983, pp. 227-250. 